\title{
A COMPARISON OF THE FORMULAS FOR THE CALCU- LATION OF THE INDUCTANCE OF COILS AND SPIRALS WOUND WITH WIRE OF LARGE CROSS SECTION
}

\author{
By Frederick W. Grover
}

ABSTRACT

Two methods have been used for the calculation of the inductance of coils of wire having a relatively large cross section. Of these, the summation method gives the inductance of the coil as the sum of the self-inductances of the turns and the mutual inductances of all the pairs of turns. The Rosa method calculates the inductance of the equivalent current sheet as a first approximation to the inductance of the coil, and obtains the correction which must be applied by calculating $(a)$ the differences between the self-inductance of the turns of wire and of the current sheet and $(b)$ the differences of the mutual inductances of pairs of turns of wire and of the corresponding turns of the current sheet.

It is here shown that, contrary to previous opinions, the two methods give identical results, when terms of the same degree are retained in the series expressions.

The accurate formula of Snow for the inductance of a helix is written so as to include the Rosa correction terms, and it is proved that the error of the Rosa method may be neglected in all except the most precise work.

It is recommended that, lacking precision formulas, the Rosa method be used as giving a general solution of the problem in such cases where the current sheet formula is known. Certain important cases are reviewed briefly.

\section{CONTENTS}

I. Introduction

II. Equivalence of the summation method and the Rosa method for solenoids of round wire

III. Summation formulas for the inductance of polygonal solenoids and spirals and their generalization

IV. Current sheet formulas for polygonal solenoids and spirals and the Rosa correction terms.

V. Recalculation of the table of the Rosa B correction

VI. Limitations of the summation method and the Rosa method....--

VII. Calculation of the inductance of a helix of round wire by Rosa's method compared with the calculation by Snow's formula......-

VIII. Generalization of the Rosa method for solenoids wound with wire of any cross section.

IX. Comparison of the Rosa method with Snow's formula for the inductance of a helix of wire of rectangular cross section

$\mathrm{X}$. Conclusion

XI. Bibliography

XII. Appendix. 


\section{INTRODUCTION}

In a recent paper Doctor Snow (1) ${ }^{1}$ has derived a formula for the calculation of the inductance of a helical coil wound with wire of any desired cross section, and has illustrated its use both for round wires and for wires of rectangular cross section. Snow's formula is very accurate, since no terms greater than those of the fourth power in the ratio of pitch of the winding to the radius of the winding form have been neglected. It also takes into account the helical shape of the winding, and thus allows for the effect of the axial component of the current in contributing to the magnetic field. This is true of no other existing formula, so that Snow's formula is able to serve as a standard in investigating the accuracy of other formulas for the inductance of a solenoid.

To a first approximation, the inductance of a single-layer coil or solenoid may be calculated by one of the numerous formulas for the inductance of a cylindrical current sheet (2). These give the value for the current sheet with great accuracy, and for coils wound closely with fine wire the difference in the inductance of coil and current sheet is not important. For wire of larger cross section, and for coils where the diameter of the wire is small compared with the pitch of the winding, the error due to the assumption that the coil and the cylindrical current sheet are equivalent is too large to be neglected in the light of the modern requirements of accuracy.

To obtain more accurate formulas, two general methods have been used, which may also be applied in other cases, such as, for example, polygonal single-layer coils and circular and polygonal spirals. For convenience these may be designated as $(a)$ the summation method, and $(b)$ the differential or Rosa method. In the former the inductance is found by summing the self-inductances of all the turns and the mutual inductances of all the pairs of turns of which the coil is composed. By this method, in 1905, Strasser (3) obtained a formula for the inductance of a circular single-layer coil of round wire. In more recent years Esau (4), by the use of the same method has obtained expressions for the inductance of circular flat spirals, of singlelayer coils wound on square forms, and of flat spirals with square turns, and Koga (5) has extended the method to triangular, hexagonal, and octagonal coils and spirals of round wire.

The second method was developed in 1906 by Rosa (6), who employed it to calculate the inductance of a single-layer circular coil wound with round wire, and published tables to aid in the calculations. In Rosa's method the difference is calculated between the inductance of the coil and that of a cylindrical current sheet having the same

1 The figures given in parentheses here and throughout the text relate to the reference numbers in the bibliography given in Section XI of this paper. 
mean radius, the same number of turns, and an axial length equal to the product of the number of turns and the pitch of the winding. The difference of inductance in the two cases is expressed as the sum of two terms, one of which takes into account the difference in the self-inductance of a turn of wire and that of a turn of the current sheet, while the other depends on the difference in the mutual inductance of corresponding pairs of turns of the coil and of the current sheet. These correction terms are added to the inductance of the current sheet to find the inductance of the coil. The inductance of the current sheet may be calculated by that one of the known formulas which is most suitable for the case in question.

The Rosa method is evidently capable of extension to other cases, provided that the expressions for the correction terms can be obtained. Its use for single-layer coils of round wire wound on square or rectangular forms has been treated by Niwa (7) in a very complete paper devoted principally to the derivation of current sheet formulas for these and many other related cases and to aids in their calculation.

Esau (8) has found differences between the values of the inductances of coils wound on square forms, found by Niwa's method and by the summation method, and has claimed a greater accuracy for the summation method. In commenting on Esau's criticism, Niwa (7) has pointed out numerical errors in Esau's calculations, but has failed to draw notice to the fact that Esau has incorrectly taken the dimensions of the current sheet to which the corrections given by the Rosa method are to be applied.

It is the purpose of the present paper $(a)$ to show that the Rosa method and the summation method lead to exactly the same formula if terms of the same order be retained in each, (b) to discuss the approximations made in deriving the formulas in the two methods, (c) to compare the Rosa method with Snow's formula for the inductance of a helical coil, for both round wire and rectangular wire, and to derive the expression for the error of the Rosa method in these two cases, and (d) to recommend the generalized Rosa method as giving the most simple and accurate method available in certain other cases, such as polygonal coils and spirals.

\section{EQUIVALENCE OF THE SUMMATION METHOD AND THE ROSA METHOD FOR CIRCULAR SOLENOIDS OF ROUND WIRE}

The summation method assumes the solenoid to be composed of $n$ coaxial circular rings of equal radii, spaced uniformly at a distance apart which is the pitch of the winding $g$. Thus, the axial length of the coil is $n g$. The current is assumed to get from one ring to the next by means of connections of negligible inductance; that is, 
the helicity of the actual winding is neglected, the current being assumed to flow in planes perpendicular to the axis of the coil.

The inductance of the coil is then

$$
L=n L_{1}+2 \underset{1}{n-1} M_{p}
$$

where $L_{1}$ is the inductance of a turn, and $M_{p}$ is the mutual inductance of two turns separated by a distance $p g$. Strasser used for $L_{1}$ a formula which is only approximate. Using the accurate formula for the inductance of a circular ring (9) of mean radius $a$ having a cross section of radius $\rho$,

$$
n L_{1}=4 \pi n a\left[\left(1+\frac{\rho^{2}}{8 a^{2}}\right) \log _{e} \frac{8 a}{\rho}-\frac{\rho^{2}}{24 a^{2}}-\frac{7}{4}\right]
$$

The mutual inductance $M_{p}$ is obtained by the Maxwell series formula (10) for the mutual inductance of coaxial circular filaments near together.

$$
M_{p}=4 \pi a\left[\left(1+\frac{3}{16} \frac{p^{2} g_{2}}{a^{2}}+\cdots\right) \log _{e} \frac{8 a}{p g}-\left(2+\frac{p^{2} g^{2}}{16 a^{2}}+\cdots\right)\right]
$$

and summing this over all the pairs of turns of the coil, we have to find

$$
2\left[(n-1) M_{1}+(n-2) M_{2}+\cdots+(n-p) M_{p}+\cdots+M_{n-1}\right]
$$

The resulting formula for the inductance of the coil is, neglecting terms in $\frac{\rho^{2}}{a^{2}}$,

$$
\begin{aligned}
L & =4 \pi a\left[n\left(\log _{e} \frac{8 a}{\rho}-1.75\right)+n(n-1)\left(\log _{e} \frac{8 a}{g}-2\right)-A_{1}\right. \\
& \left.+\frac{1}{8} \frac{g^{2}}{a^{2}}\left\{\left(3 \log _{e} \frac{8 a}{g}-1\right) \frac{n^{2}\left(n^{2}-1\right)}{12}-B_{1}\right\}\right]
\end{aligned}
$$

which is slightly more accurate than that given by Strasser, on account of the greater accuracy in the formula used for $L_{1}$. In this

$$
\begin{aligned}
A_{1} & =2\left[\log _{e}(n-1) !+\log _{e}(n-2) !+\cdots+\log 2 !\right] \\
B_{1} & =3\left[(n-1) 1^{2} \log _{e} 1+(n-2) 2^{2} \log _{e} 2+\cdots+(n-p) p^{2} \log _{e} p\right. \\
& \left.+\cdots+(n-1)^{2} \log _{e}(n-1)\right]
\end{aligned}
$$

Tables of the values of $A_{1}$ and $B_{1}$ were given by Strasser (3), including $n=30$. These were apparently calculated directly from the defining formulas (6), a tedious process.

To calculate the inductance by the Rosa method the inductance $L_{s}$ of a cylindrical current sheet of $n$ turns of radius $a$ and having a length $b=n g$ is calculated. To this is to be added the difference between the inductance of the coil and the current sheet. To obtain this difference the inductance $l_{w}$ of a turn of the round wire is cal- 
culated by formula (2), divided by $n$. The inductance $l_{t}$ of a turn of the current sheet is obtained by placing $b=g$ in the Rayleigh and Niven expression (11) for the inductance of a short cylindrical current sheet. (See Formula (15) below.) Subtracting $l_{t}$ from $l_{w}$ and multiplying by $n$, the difference in inductance of coil and current sheet due to the fact that the individual turns of wire have a different

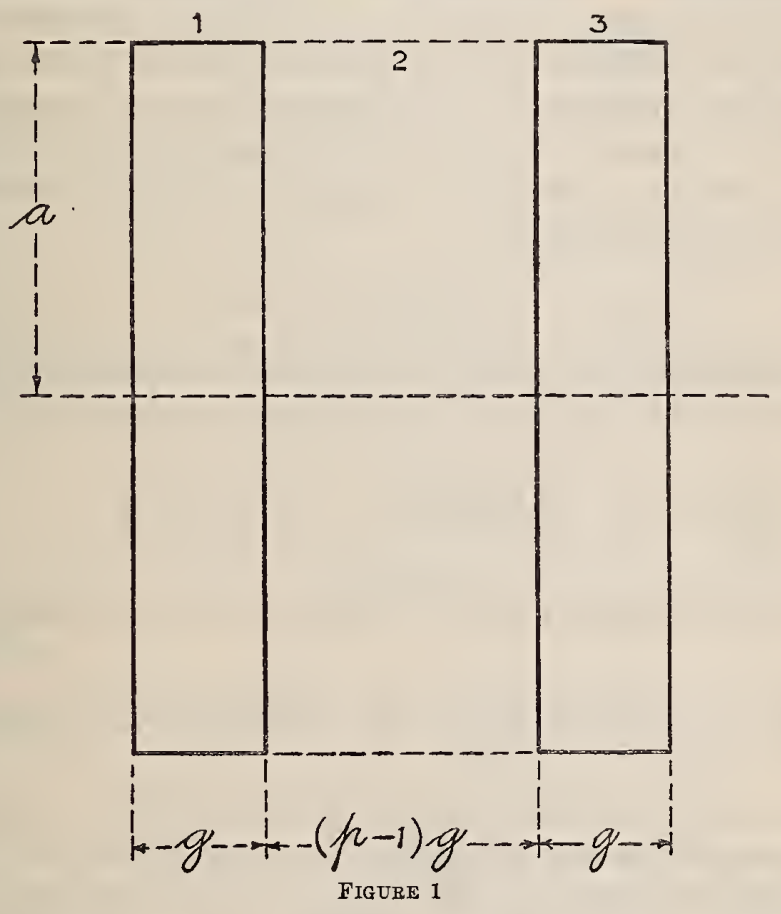

inductance than the turns of the current sheet is obtained. The result is, neglecting terms in $\rho^{2} / a^{2}$,

$$
n\left(l_{w}-l_{t}\right)=4 \pi a\left[n\left(\log _{e} \frac{g}{\rho}-\frac{5}{4}\right)-\frac{n g^{2}}{32 a^{2}}\left(\log _{e} \frac{8 a}{g}+\frac{1}{4}\right)\right]=-4 \pi n a A
$$

The principal term of this is the constant $A$ of Rosa, only with opposite sign. It will be convenient to speak of this type of correction as the " $A$ correction."

Designating by $M_{p}$ the mutual inductance of any pair of turns of the wire separated by a distance of $p g$, and by $m_{p}$ the mutual inductance of the corresponding turns of the current sheet, then the total correction to the inductance of the current sheet due to all the pairs of wire is

$$
\begin{aligned}
2\left[(n-1)\left(M_{1}-m_{1}\right)\right. & +(n-2)\left(M_{2}-m_{2}\right)+\cdots+(n-p)\left(M_{p}-m_{p}\right)+\cdots \\
& \left.+\left(M_{n-1}-m_{n-1}\right)\right]=-4 \pi n a B
\end{aligned}
$$


For convenience this will be referred to as the "B correction." The inductance of the coil is then

$$
L=L_{s}-4 \pi n a(A+B)
$$

The values of $M_{p}$ are to be calculated by the Maxwell expression for circular filaments, formula (3). To obtain $m_{p}$ we have to find the mutual inductance of the two equal short cylindrical sheets, 1 and 3 , Figure 1, whose lengths are $g$ and whose central planes are separated by a distance $p g$. Supposing the space between them to be filled by a current sheet (2) of the same radius and wound with the same pitch, its axial length being $(p-1) g$, the required mutual inductance will be given by

$$
2 m_{p}=L_{123}+L_{2}-2 L_{12}
$$

The self-inductances in this formula may be obtained from the current sheet formula (15) below, and the resulting expression is

$$
\begin{aligned}
m_{p} & =4 \pi a\left[\left(\log _{e} 8 a-\frac{1}{2}\right)+\frac{g^{2}}{32 a^{2}}\left(6 p^{2}-1\right)\left(\log _{e} \frac{8 a}{g}+\frac{1}{4}\right)\right. \\
& -\left\{\frac{(p+1)^{2}}{2} \log _{e}(p+1) g+\frac{(p-1)^{2}}{2} \log _{e}(p-1) g-p^{2} \log _{e} p g\right\} \\
& \left.-\frac{g^{2}}{64 a^{2}}\left\{(p+1)^{4} \log _{e}(p+1)+(p-1)^{4} \log _{e}(p-1)-2 p^{4} \log _{e} p\right\}\right]
\end{aligned}
$$

The second line of this formula differs only by a constant term from the expression for the geometric mean distance $R_{p}$ of the two straight lines of length, $g$, which form the cross sections of the two turns of the current sheet (13). Writing $-Y$ for the last line of equation (11), and subtracting equation (11) from equation (3) there is found the general relation

$$
\begin{aligned}
M_{p}-m_{p} & =4 \pi a\left[\log _{e} \frac{R_{p}}{p g}-\frac{3}{16} \frac{p^{2} g^{2}}{a^{2}} \log _{e} p-\frac{g^{2}}{32 a^{2}} \log _{e} \frac{8 a}{g}\right. \\
& \left.-\frac{7 p^{2} g^{2}}{64 a^{2}}-\frac{g^{2}}{128 a^{2}}+Y\right]
\end{aligned}
$$

which is to be summed over the coil according to equation (8).

Writing for $\log \frac{R_{p}}{g}$ its value (12)

$$
\log _{e} \frac{R_{p}}{g}=\frac{(p+1)^{2}}{2} \log _{e}(p+1)+\frac{(p-1)^{2}}{2} \log _{e}(p-1)-p^{2} \log _{e} p-\frac{3}{2}
$$

and summing over the coil there is found $n^{2} \log _{e} n-\frac{3}{2} n(n-1)$, while 
the summation of $\log p$ gives the constant $A_{1}$ of Strasser, defined in equation (6). Thus we may identify the constant $B$ of Rosa with the expression

$$
n \log _{e} n-\frac{3}{2}(n-1)-\frac{A_{1}}{n}=-\bar{B}
$$

in which $\bar{B}$ has been put for the Rosa constant, which is the principal term of $B$ in equation (8).

The summation of the second term in equation (12) leads to $-\frac{1}{8} \frac{g^{2}}{a^{2}} B_{1}$, where $B_{1}$ is Strasser's constant, formula (6), while the summation of $Y$ yields $\frac{n^{4} g^{2}}{32 a^{2}} \log _{e} n$ only.

In order to compare Rosa's method with the summation method, the current sheet inductance $L_{s}$ will here be expressed by the Rayleigh and Niven's formula (11) for a short cylindrical current sheet of radius $a$, length $b$,

$$
L=4 \pi a n^{2}\left[\left(\log _{e} \frac{8 a}{b}-\frac{1}{2}\right)+\frac{b^{2}}{32 a^{2}}\left(\log _{e} \frac{8 a}{b}+\frac{1}{4}\right)-\cdots\right]
$$

although no such limitation is inherent in the method. Thus writing $n g$ for $b$ in equation (15) the terms in equation (9) may be collected and we have

$$
\begin{gathered}
L_{s}=4 \pi a\left[n^{2}\left(\log _{e} \frac{8 a}{n g}-\frac{1}{2}\right)+\frac{n^{4} g^{2}}{32 a^{2}}\left(\log _{e} \frac{8 a}{n g}+\frac{1}{4}\right)\right] \\
-4 \pi n a A=4 \pi a\left[n\left(\log _{e} \frac{g}{\rho}-\frac{5}{4}\right)-\frac{n g^{2}}{32 a^{2}}\left(\log _{e} \frac{8 a}{g}+\frac{1}{4}\right)\right] \\
-4 \pi n a B=4 \pi a\left[n^{2} \log _{e} n-\frac{3}{2} n(n-1)-A_{1}-\frac{1}{8} \frac{g^{2}}{a^{2}} B_{1}-\frac{n(n-1)}{128} \frac{g^{2}}{a^{2}}\right. \\
\left.-\frac{n(n-1)}{32} \frac{g^{2}}{a^{2}} \log _{e} \frac{8 a}{g}-\frac{7}{384} \frac{n^{2}\left(n^{2}-1\right) g^{2}}{a^{2}}+\frac{n^{4} g^{2}}{32 a^{2}} \log _{e} n\right]
\end{gathered}
$$

Adding and simplifying

$$
\begin{gathered}
L=4 \pi a\left[n^{2}\left(\log _{e} \frac{8 a}{g}-2\right)+n\left(\log _{e} \frac{g}{\rho}+\frac{1}{4}\right)-A_{1}-\frac{1}{8} \frac{g^{2}}{a^{2}} B_{1}\right. \\
\left.+\frac{n^{2}\left(n^{2}-1\right)}{32} \frac{g^{2}}{a^{2}} \log _{e} \frac{8 a}{g}-\frac{n^{2}\left(n^{2}-1\right)}{96} \frac{g^{2}}{a^{2}}\right]
\end{gathered}
$$

and if we add and subtract $\left(n \log _{e} \frac{8 a}{g}+2 n\right)$, the expression goes over into Strasser's formula (5) exactly.

This result was to have been expected, since the methods should agree if correct inductance formulas are used, and if, in both methods, terms of the same order are retained. 
In the correction constants $A$ and $B$, as given by Rosa, terms in $\frac{g^{2}}{a^{2}}$ were neglected as of small effect, compared with the main or geometric mean distance terms. This was justified by Rosa by numerical examples, but it may be shown more generally that the terms in $\frac{g^{2}}{a^{2}}$ in equation (17) are very small. To accomplish this, the Strasser constant $B_{1}$ may be expanded in the asymptotic series

$$
B_{1}=\frac{1}{4}\left(n^{4}-n^{2}+\frac{1}{10}\right) \log _{e} n-\frac{7}{48} n^{4}+\frac{n}{12}\left(1+\frac{1}{10}-\cdot .\right)+\frac{1}{16}+\frac{1}{1008 n^{2}}+\cdots
$$

and substituting this in the last two equations of (16) it is found that the combined terms in $\frac{g^{2}}{a^{2}}$ are $-\frac{n^{2} g^{2}}{32 a^{2}}\left(\log _{e} \frac{8 a}{n g}-\frac{1}{3}\right)-\frac{1}{96} \frac{n g^{2}}{a^{2}}-$ smaller terms. For the case $n=30, g=0.1, a=15$, these terms amount to only 8 parts in 10,000 of the total correction, and the latter is only 18 parts in 10,000 of the whole inductance of the coil, so that the neglected terms here are of the order of only a part in a million of the whole inductance. A further discussion of this point will be made in Section VI.

\section{SUMMATION FORMULAS FOR THE INDUCTANCE OF POLYGONAL SOLENOIDS AND SPIRALS AND THEIR GENERALIZATION}

Formulas for the inductance of polygonal solenoids and spirals have been obtained by Esau (4) and by Koga (5). Esau treated the case of square coils, making use of the known formulas for the inductance of a square of round wire and for the mutual inductance of equal parallel coaxial square filaments.

Koga extended the method to triangular, hexagonal, and octagonal coils and spirals, and derived for this purpose the basic formulas which had to be summed over the coil. Thus he gives formulas for the mutual inductance of concentric coplanar polygons with their sides parallel. His formulas for the inductance of polygons of round wire and for the mutual inductance of equal parallel coaxial polygons check those of the author of the present paper which were published a little earlier (13).

Both Esau and Koga in their use of the summation method first expanded the basic formulas in terms of the ratio of the pitch to the length of a side of the polygon. Tables are given for different numbers of turns up to 30 , and for different sizes of wire and pitch of winding. Since powers no higher than the square of the ratio of pitch to side of polygon are retained, the formulas converge well only for relatively short coils or for spirals of small axial width. 
An inspection of these summation formulas has made clear that they may all be written in the same form, only with different numerical coefficients depending upon the number of sides of the polygon. After checking all the coefficients and correcting errors in two instances, ${ }^{2}$ the writer finds the following results, the constants being collected in Tables 1 and 2 .

Inductance of a polygon of round wire.

in which

$$
L=2 N a\left[\log _{e} \frac{a}{\rho}+q\right]
$$

$N=$ the number of sides of the polygon,

$a=$ the length of a side of the polygon,

$\rho=$ the radius of cross section of the wire.

Mutual inductance of equal parallel coaxial polygons.

$$
M=2 N a\left[\log _{e} \frac{a}{d}-r+s \frac{d}{a}+t \frac{d^{2}}{a^{2}}\right]
$$

where $d=$ the distance between their planes.

Inductance of polygonal solenoids.

Letting $n$ be the number of turns, $g$ the pitch of winding

$$
\begin{aligned}
L= & 2 \operatorname{Nan}\left[\left(\log _{e} \frac{a}{\rho}+q\right)+(n-1)\left(\log _{e} \frac{a}{g}-r\right)\right. \\
& \left.+\frac{n^{2}-1}{3} \cdot s \frac{g}{a}+\frac{n\left(n^{2}-1\right)}{6} \cdot t \frac{g^{2}}{a^{2}}-\frac{A_{1}}{n}\right]
\end{aligned}
$$

\begin{tabular}{|c|c|c|c|c|}
\hline . & $q$ & $r$ & $s$ & $t$ \\
\hline $\begin{array}{l}\text { Triangles.- } \\
\text { Squares.- } \\
\text { Hexagons } \\
\text { Octagons.- }\end{array}$ & $\begin{array}{l}-1.1555 \\
-.52401 \\
+.098476 \\
+.46198\end{array}$ & $\begin{array}{r}1.4055 \\
.77401 \\
-.15152 \\
-.21198\end{array}$ & $\begin{array}{l}2.2092 \\
1 \\
.3954 \\
.2146\end{array}$ & $\begin{array}{r}-13 / 12 \\
-0.0429 \\
+.1160 \\
+.1052\end{array}$ \\
\hline
\end{tabular}

$A_{1}$ is Strasser's constant, formula (6).

TABLE 1.-Values of numerical constants in formulas (19), (20), (21), and (22)

Mutual inductance of parallel concentric coplanar polygons.

Putting here $a$ for the mean length of a side of the polygon

$$
M=2 N a\left[\log _{e} \frac{a}{d}-r+s^{\prime} \frac{d}{a}+t^{\prime} \frac{d^{2}}{a^{2}}\right]
$$

the constants being given in Table 2, excepting $r$ which is the same as in the preceding formulas

.2 Koga gives $S^{1}$ for the octagon as $-(\sqrt{2}-1) \times 8.5509=-0.3452$ and $T$ for the triangle as $\frac{13}{12}$. 
Inductance of a polygonal flat spiral.

If $a$ represents the mean length of a side of the polygonal turns of the spiral, $g$ the (axial) pitch of the winding, $n$ the number of turns

$$
\begin{aligned}
L=2 & \operatorname{Nan}\left[\left(\log _{e} \frac{a}{\rho}+q\right)+(n-1)\left(\log _{e} \frac{a}{g}-r\right)\right. \\
& \left.+\frac{n^{2}-1}{3} s^{\prime} \frac{g}{a}+\frac{n\left(n^{2}-1\right)}{6} \frac{T g^{2}}{a^{2}}-\frac{A_{1}}{n}\right]
\end{aligned}
$$

Between the coefficient $T$ and $t^{\prime}$ there exists the relation $T=t^{\prime}-\frac{v^{2}}{2}$. The constants of the last two formulas are collected in Table 2.

\begin{tabular}{|c|c|c|c|c|}
\hline & $s^{\prime}$ & $T$ & $t^{\prime}$ & 0 \\
\hline Triangles_- & \multirow{3}{*}{$\begin{array}{l}1.2396 \\
.53284 \\
.20328 \\
.10898\end{array}$} & \multirow{3}{*}{$\begin{array}{c}23 / 12 \\
3 / 4 \\
0.2947 \\
.1662\end{array}$} & \multirow{3}{*}{$\begin{array}{l}5 / 13 \\
1 / 4 \\
0.1280 \\
.0804\end{array}$} & \multirow{3}{*}{$\begin{array}{r}\sqrt{3} \\
1 \\
1 / \sqrt{3} \\
(\sqrt{2}-1)\end{array}$} \\
\hline $\begin{array}{l}\text { Squares-- } \\
\text { Hexagons.----- }\end{array}$ & & & & \\
\hline Octagons_-_--.-- & & & & \\
\hline
\end{tabular}

TABLE 2.-Values of numerical constants in formulas (22) and (23)

IV. CURRENT SHEET FORMULAS FOR POLYGONAL SOLENOIDS AND SPIRALS AND THE ROSA CORRECTION TERMS

The formula for the inductance for a solenoidal current sheet on a square form was derived by Niwa (14), and independently, at about the same time, by the writer (13), who also derived series formulas for short triangular, hexagonal, and octagonal current sheets.

To obtain the formula for the inductance of a polygonal solenoid by the Rosa method, it is necessary to start with the formula for the corresponding current sheet. Putting, as before, $a$ for the mean side of the polygon, and supposing that the $n$ turns have an axial length of $n g$, the generalized formula (13) for the inductance of the current sheet is

$$
L_{s}=2 \operatorname{Nan}^{2}\left[\log _{e} \frac{a}{n g}+\left(\frac{3}{2}-r\right)+\frac{s}{3} \frac{n g}{a}+\frac{t}{6} \frac{n^{2} g^{2}}{a^{2}}\right]
$$

which holds for cases where the ratio of the axial length to the side of the polygon is small. The numerical coefficients have the values given in Table 1.

The Rosa corrections for the cross section of an actual winding of round wire may be obtained by the method already outlined for a circular solenoid in Section II. The $A$ correction is obtained from equation (24), putting $g$ in place of $n g$. This gives the inductance of a turn of the current sheet. Formula (19) gives the inductance of a turn of the round wire. The $B$ correction is obtained by summing over the coil an equation corresponding to equation (8), the values 
of the mutual inductances for two turns of the current sheet being obtained from differences of self-inductances, as in equation (10), using equation (24), while the values for pairs of turns of the round wire are given by equation (20) for polygonal filaments.

The results may be summarized as follows:

$$
\begin{aligned}
L_{s} & =2 \operatorname{Nan}^{2}\left[\log _{e} \frac{a}{n g}+\left(\frac{3}{2}-r\right)+\frac{s}{3} \frac{n g}{a}+\frac{t}{6} \frac{n^{2} g^{2}}{a^{2}}\right] \\
-2 \operatorname{Nan} A & =2 \operatorname{Nan}\left[\log _{e} \frac{g}{\rho}-\left(\frac{3}{2}-r\right)+q-\frac{s}{3} \frac{g}{a}-\frac{t}{6} \frac{g^{2}}{a^{2}}\right] \\
-2 N a n B & =2 \operatorname{Nan}\left[n \log _{e} n-\frac{3}{2}(n-1)-\frac{A_{1}}{n}-\frac{t}{6}(n-1) \frac{g^{2}}{a^{2}}\right]
\end{aligned}
$$

and adding these, the inductance of the polygonal solenoid is

$$
\begin{gathered}
L=2 \operatorname{Nan}\left[\left(\log _{e} \frac{a}{\rho}+q\right)+(n-1)\left(\log _{e} \frac{a}{g}-r\right)-\frac{A_{1}}{n}+\frac{n^{2}-1}{3} \frac{s g}{a}\right. \\
\left.+\frac{n\left(n^{2}-1\right)}{6} \frac{t g^{2}}{a^{2}}\right]
\end{gathered}
$$

which is, exactly, the formula (21) obtained by the summation method for this case.

Niwa (7) calculated the correction for cross section as equal to twice the total length of wire in the coil, times the sum of the two constants tabulated by Rosa for circular solenoids, and applied it to his current sheet formulas for square and rectangular solenoids. This procedure is justified, if we neglect terms in $\frac{g}{a}$ and $\frac{g^{2}}{a^{2}}$, since it will be found that, using the values of Table 1 , the quantity $-\left(\frac{3}{2}-r\right)+q$ is always equal to $-\frac{5}{4}$, whatever the number of sides of the polygon. Thus, comparing with equation (16) the principal terms of the correction are $-2 \operatorname{Nan}(A+B)$, the constants $A$ and $B$ being the same as for a circular solenoid.

To treat the polygonal spiral by the same method requires a current sheet formula for a polygonal disk or ring, and no formula for this case has been published. It may readily be derived by the method of geometric mean distance and arithmetical mean distances by substituting in formula (22) for coplanar polygons, $\log n g-\frac{3}{2}$ for $\log d$, $\frac{1}{3} n g$ for $d(15)$, and $\frac{1}{6} n^{2} g^{2}$ for $d^{2}$. This result was checked by integrating equation (22) twice over the current sheet. This yields a formula which differs from that obtained by the simpler method only in the coefficient of $\frac{n^{2} g^{2}}{a^{2}}$, the simpler method giving $t^{\prime}$ instead of $T$. 
The value by integration, which is the more accurate result, may be written in the general form

$$
L_{s}=2 N n^{2} a\left[\log _{e} \frac{a}{n g}+\left(\frac{3}{2}-r\right)+q+\frac{s^{\prime}}{3} \frac{n g}{a}+\frac{T}{6} \frac{n^{2} g^{2}}{a^{2}}\right]
$$

in which $a$ is the side of the mean polygonal filament. The coefficients have already been given in Tables 1 and 2 .

The derivation of the Rosa corrections for cross section follows the course already illustrated in Section II, although the process of summation is more involved, on account of the varying sizes of the turns of the spiral.

The resulting correction terms are

$$
\begin{aligned}
& -2 \operatorname{Nan} A=2 \operatorname{Nan}\left[\log _{e} \frac{g}{\rho}+q-\left(\frac{3}{2}-r\right)-\frac{s^{\prime}}{3} \frac{g}{a}-\frac{T}{6} \frac{g^{2}}{a^{2}}\right] \\
& -2 \operatorname{Nan} B=2 \operatorname{Nan}\left[n \log _{e} n-\frac{3}{2}(n-1)-\frac{A_{1}}{n}-\frac{T}{6}(n-1) \frac{g^{2}}{a^{2}}\right]
\end{aligned}
$$

and adding these to the current sheet value in equation (26), the final formula for the polygonal spiral is exactly the same as formula (23), which was derived by the summation method. Here also as in the previous case the principal terms of the correction have the same value, and may be calculated from the same table of constants as for circular solenoids.

It has been proved that the Rosa method and the summation method agree, if terms of the same degree are retained in both, not only in the case of circular solenoids but also with polygonal solenoids and spirals. It may be shown by the same methods that this result is likewise true for circular flat spirals. As already intimated, these results were to have been expected, and that they are found to be true gives a check on the various inductance formulas employed. It also shows that Esau's criticism is without ground.

\section{RECALCULATION OF THE TABLE OF THE B CORRECTION OF ROSA}

The quantity $B$ tabulated by Rosa for wire of round cross section was defined by the relation (6)

$$
\begin{gathered}
B=\frac{2}{n}\left[(n-1) \log _{e} \frac{R_{1}}{g}+(n-2) \log _{e} \frac{R_{2}}{2 g}+\cdots+(n-p) \log _{e} \frac{R_{p}}{p g}+\cdots\right. \\
\left.+\log _{e} \frac{R_{n-1}}{(n-1) g}\right]
\end{gathered}
$$

in which $\frac{R_{p}}{p g}$ represents the ratio of the geometric mean distance of two straight lines (cross sections of a pair of the turns of the current 
sheet) whose centers are separated by a distance $p g$, to the distance between them. This quantity may be calculated by formula (130) of Bureau of Standards Scientific Paper No. 169, and for more distant turns by the very convergent series formula (131) of the same paper. Thus the direct calculation of $B$ by formula (28) offers no difficulty, except that the calculation for large values of $n$ becomes very tedious. Furthermore, in the calculation of a table for different values of $n$, the fact that the calculation for a given value of $n$ rests upon the calculation for smaller values of $n$, although seemingly an advantage, works to the end that any error made with a smaller value of $n$ is carried through into the calculation for the larger values of $n$. Thus, it is difficult to obtain values of $B$ for the larger values of $n$ which shall be free from error.

From equations (8) and (12) (the first term), it has already been noted that the correction $B$ may be written in the form shown in equation (14). This equation, which does not seem to have been previously noticed, gives a means for checking the values found by the formula (28). For this purpose the table of values of $A_{1}$, given by Strasser and, corrected for small errors, in Table 5, Bureau of Standards Scientific Paper No. 169, should be useful. Beyond $n=30$, the range of this table, the quantity $A_{1}$, as may be seen from equation (6), labors under the same disadvantage for purposes of calculation as does equation (28).

This difficulty may be very completely avoided by using for $A_{1}$ a development in an asymptotic series, as was done by Rolf (16) and by Koga (5). Since, however, the expressions used by these two authors differ slightly, it was necessary to investigate the cause for the discrepancy. The two expressions are as follows:

$$
\begin{aligned}
& \frac{A_{1}}{n}=n\left(\log _{e} n-\frac{3}{2}\right)+\log _{e} 2 \pi-\frac{1}{6 n} \log _{e} n-\frac{0.3312}{n}-\frac{1}{120 n^{3}}+\frac{7}{2160 n^{4}} \text { (Rolf) } \\
& \frac{A_{1}}{n}=n\left(\log _{e} n-\frac{3}{2}\right)+\log _{e} 2 \pi-\frac{1}{6 n} \log _{e} n-\frac{0.33084}{n}-\frac{1}{120 n^{3}}+\frac{1}{270 n^{4}} \text { (Koga) }
\end{aligned}
$$

Koga explains that his expression was derived by applying the Euler (17) summation formula to the Stirling asymptotic formula for $\log m$ !, making use, of course, of the defining equation for $A_{1}$, formula (6). The Stirling formula is (17)

$$
\log _{e} m !=\left(m+\frac{1}{2}\right) \log _{e} m-m+\frac{1}{2} \log _{e} 2 \pi+\frac{1}{12 m}-\frac{1}{360 m^{3}}+\frac{1}{1260 m^{5}}
$$

This may be summed directly by formula (6) for integral values of $m$ from 1 to $(n-1)$, inclusive, the series which enter being made to depend upon known series, or it may be summed by applying the $53811^{\circ}-29-12$ 
Euler summation formula to each term. The result is an asymptotic series in which the term in $\frac{1}{n}$ is given by an asymptotic series, while the coefficients of the terms in higher powers of $\frac{1}{n}$ begin to increase after that of $\frac{1}{n^{5}}$. The numerical coefficient of $\frac{1}{n}$ was found to be about -0.33086 , but, by making use of the exact formula for $A_{1}$, it is found that, for all values of $n$ greater than 3 , the value -0.330842 is indicated as correct. Adopting this value the resulting expansion is

$\frac{A}{n}=n\left(\log _{e} n-\frac{3}{2}\right)+\log _{e} 2 \pi-\frac{1}{6 n} \log _{e} n-\frac{0.330842}{n}-\frac{1}{120 n^{3}}+\frac{1}{504 n^{5}}$

which differ from Koga's expression only in the last term. This difference is of no consequence for all except the smallest values of $n$. (Evidence will be given later of the correctness of the last term of equation (30).)

Substituting equation (30) in equation (14) there results

$$
B=0.337877-\frac{1}{6 n} \log _{e} n-\frac{0.330842}{n}-\frac{1}{120 n^{3}}+\frac{1}{504 n^{5}}
$$

The first term $\left(\log 2 \pi-\frac{3}{2}\right)$ shows what is the limiting value of $B$, as $n$ increases indefinitely, a value which has hitherto been lacking. Formula (31) has been checked for values of $n$ up to 30 , by calculating $A_{1}$ directly from equation (6), and also by making use of Strasser's values. For all values of $n$ greater than 3 , it is found that equation (31) gives a 6-figure accuracy or better. The error is evidently smaller, the greater the value of $n$; that is, the formula is most accurate in just those cases where the exact formula is most difficult to calculate.

By these means Table 8, Bureau of Standards Scientific Paper No. 169 , has been recalculated. The values of $B$ were found to be correct up to $n=15$, but beyond that point the values of Table 8 are too large by amounts which vary from a few units up to 12 units in the fourth place. The revised table is given as Table 3 in the Appendix.

\section{LIMITATIONS OF THE SUMMATION METHOD AND THE ROSA METHOD}

In the preceding sections it has been proved that the summation method and the Rosa method lead to exactly the same expressions for the inductance of solenoids and spirals, when terms of the same order are retained in both. The proof has included second degree terms only, but there is no reason to doubt that the agreement would 
be found for higher degree terms in the series expansions. The agreement is, in fact, only a check on the basic inductance formulas which have been employed in the two methods. This agreement of the two methods does not signify, however, that the Rosa method and summation method are of equal accuracy in all cases.

Since in order to be able to carry out the summations with any degree of simplicity, it is necessary in the summation method to use series expansions for the basic formulas which are to be summed, it follows that the summation method gives series formulas which converge well only for short coils or narrow spirals. Thus the tables of Strasser, Esau, and Koga cover a range up to only 30 turns of wire.

This limitation is not inherent in the Rosa method. It is true that in the comparison of the Rosa method with the summation method the current sheet formulas used were series expansions, subject to the same limitations as the summation formulas, but these were used merely for the purpose of proving the identity of the final results by both methods. Current sheet formulas are, however, available for circular solenoidal current sheets, and for circular disks which fit all cases and give an accuracy greater than is necessary in practice, and to the suitable current sheet formula in any given case the Rosa correction may be applied. It remains only to show that the Rosa correction may be calculated with an accuracy sufficient to cause no appreciable error in the final result.

Since the correction to the current sheet value of the inductance to take into account the cross section of the actual winding is usually no greater than about 1 per cent of the whole inductance, the correction does not need to be calculated with great accuracy in order to give a suitable accuracy in the total inductance. As originally developed by Rosa, only the principal or geometric mean distance terms were included, and it will be shown that these terms, which we may conveniently designate as the "simple Rosa corrections," are, in general, sufficient.

Consider, for example, two turns of a circular solenoid of round wire and the corresponding two turns of the current sheet, which has the same radius and pitch of winding. The mutual inductance of the two turns of wire may be calculated by formula (3) in which the terms in $\frac{p^{2} g^{2}}{a^{2}}$, which become zero for circles of very great radius $a$, may be regarded as corrections for the curvature of the turns. Likewise for the turns of the current sheet, curvature terms enter, as is shown by equation (11). In both cases the curvature terms are not negligible, but in the difference of the two mutual inductances, which is the quantity which enters into the Rosa correction, the curvature terms must nearly cancel, unless the dimensions of the cross sections 
are large in comparison with the radius of the turns. It has already been shown that these differences of curvature terms are, for a short coil, of very small effect on the Rosa correction terms. (See p. 170.)

For a long coil the total effect of the curvature terms is not so easy to evaluate, since they are expressed by a series which converges only for relatively short coils. It is evident also that, for the more distant turns of wire, the curvature terms are relatively more important compared with the principal terms. However, the mutual inductances of the distant turns, and still more so their differences, are very small, so that the curvature effect for such turns should contribute, absolutely, very little to the total correction.

The following detailed examination of a practical problem will make some of these points clearer, in that it will give a more quantitative measure of the importance of the terms neglected. The coil considered has 400 turns, wound on a form of $15 \mathrm{~cm}$ radius with a winding pitch of $0.1 \mathrm{~cm}$, so that the coil has an axial length of $40 \mathrm{~cm}$. The contribution to the Rosa $B$ correction of all the turns which are separated by a distance of $p g$ is $-4 \pi a\left[2(n-p) \log _{e} \frac{R_{p}}{p g}\right]$ The contributions to the correction for pairs of turns of all distances up to $p=10$ are as follows:

\begin{tabular}{rrrrrr}
$p$ & Correction & $p$ & Correction & $p$ & \multicolumn{1}{c}{ Correction } \\
1 & $-4 \pi a(90.741)$ & 4 & $-4 \pi a(4.178)$ & 7 & $-4 \pi a(1.342)$ \\
2 & $(17.496)$ & 5 & $(2.655)$ & 8 & $(1.024)$ \\
3 & $(7.522)$ & 6 & $(1.834)$ & 9 & $(0.806)$
\end{tabular}

giving a total of $-4 \pi a(127.598)$. The total sum of the principal terms may be obtained from Table 3 for $n=400$. It is $-4 \pi a[400 \times$ $0.33455]=-4 \pi a(133.820)$; that is, the contributions of all the pairs of turns separated by more than nine times the pitch is only about 5 per cent of the whole $B$ correction. It may be shown that turns for which $p$ is greater than 20 contribute only 2.2 per cent, those at distances greater than $50 \mathrm{~g}$ only 0.6 of 1 per cent, while those for which the separation is greater than $250 \mathrm{~g}$ account for only 1 part in 6,500 of the whole.

For separations as great as $3 g$ the contribution of all the pairs of turns with a separation $p g$ may be calculated from Rosa's (19) formula (49), Bureau of Standards Scientific Paper No. 169, which reads:

$$
\begin{gathered}
\Delta M_{p}=-4 \pi a \cdot 2(n-p)\left[\frac { 1 } { 1 2 } \frac { 1 ^ { 2 } } { p ^ { 2 } } \left\{1+\frac{3}{8} \frac{p^{2} g^{2}}{a^{2}}\left(\log _{e} \frac{8 a}{p g}-\frac{11}{6}\right)-\frac{45}{256} \frac{p^{4} g^{4}}{a^{4}}\right.\right. \\
\left.\left.\left(\log _{e} \frac{8 a}{p g}-\frac{97}{60}\right)+\cdot \cdot\right\}+\frac{1}{60} \frac{1}{p^{4}}\left\{1+\frac{1}{16} \frac{p^{2} g^{2}}{a^{2}}\right\}+\frac{1}{168} \frac{1}{p^{6}}+\cdot\right]
\end{gathered}
$$

In this formula the series in powers of $\frac{1}{p^{2}}$ is the expansion of the 
geometric mean distance ratio; the other terms give, therefore, the curvature terms. For cases where formula (32) converges, the summation of these terms can be performed, and their effect evaluated, as was done for a winding of 30 turns on the same form, page 170 . For the long coil here considered, formula (32) is not sufficiently convergent in the case of turns which are separated by distances much greater than the radius of the coil. In such cases the Rosa-Weinstein (19) formula (50) of Bureau of Standards Scientific Paper No. 169 may be used in place of formula (32) to obtain the contributions of chosen pairs of wires. Thus, for the extreme turns, for which $p=399$, the true value of $\Delta M_{p}$ is $-4 \pi a\left[2.3 \times 10^{-7}\right]$, while the principal terms of formula (32) give a value which is numerically more than twice as great. The 150 different pairs of turns separated by a distance of $25 \mathrm{~cm}$ contribute $\Delta M_{p}=-4 \pi a[0.000331]$, while the $g, m, d$ terms are about 20 per cent greater. Unfortunately, since the RosaWeinstein formula involves elliptical integrals, it is impracticable to obtain its summation over the coil. It is, however, evident that, for the more distant turns of the coil, the curvature effect is of opposite sign to what it is for the nearer turns, so that, proportionately, the curvature effect should be smaller rather than greater than for a short coil on the same form, and it has already been shown that in the latter case its effect on the whole inductance is very small.

The summation method of Strasser assumes that the current flows in circular turns whose planes are perpendicular to the axis of the solenoid. Thus the effect of the axial component of the current, which is present when the current flows in the actual helical winding, is neglected. The effect of the axial component has also been neglected in obtaining the correction for cross section by the Rosa method, but if a current sheet formula for a true continuous helix without insulation be available, the neglect of helicity in getting the correction for cross section is of second order in its effect on the total inductance. In a recent paper Snow (1) has given a very accurate formula for the inductance of a helix of wire, which enables the correction to be calculated which must be applied to the formula for a cylindrical current sheet to obtain the true inductance of the helix. He gives also the formula for the inductance of a true continuous helical current sheet. Thus, it is now possible to evaluate the error from neglecting the helicity of the winding, and also to calculate what is the error of the simple Rosa method when applied to the calculation of the inductance of a helical winding of wire of round or rectangular cross section. 


\section{CALCULATION OF THE INDUCTANCE OF A HELIX OF ROUND WIRE BY ROSA'S METHOD COMPARED WITH THE CALCULATION BY SNOW'S FORMULA}

The formula for the inductance of a helix of round wire is given as equation (114), page 466 , in Snow's article. ${ }^{3}$ In the nomenclature of the present paper this reads

$$
\begin{gathered}
L=L_{o}+4 \pi n a\left[-\left(0.89473-\log _{e} \frac{g}{2 \rho}\right)+\frac{1}{6 n} \log _{e} \frac{2 \pi a}{g}+\frac{A_{2}(k)}{2 n}\right. \\
\left.+\frac{1}{4 n}\left(\frac{E}{k}-1\right)\left(\frac{2 \rho}{g}\right)^{2}\right]
\end{gathered}
$$

In this $L_{o}$ is the inductance of a cylindrical current sheet (helicity neglected) whose length is $n g$ and whose mean radius is $a, k=\frac{2 a}{\sqrt{4 a^{2}+n^{2} g^{2}}}$ is the modulus of the elliptic integral of second kind $\mathrm{E}$, and $A_{2}(k)$ is a complicated function of $k$, to be obtained from the curve of page 475. It is the principal term in the correction for the effect of the axial component of the current.

If we notice that $0.89473=\log \pi-\frac{1}{4}$, and substitute for $\log 2 \pi-\frac{3}{2}$ its value in terms of $B$ from equation (31), we find, making use of equation (7), that Snow's formula may be written for uniform current distribution

$$
\begin{aligned}
L=L_{o}+ & 4 \pi n a\left[-(A+B)-\frac{1}{6 n} \log _{e} \frac{n g}{a}-\frac{0.02458}{n}+\frac{A_{2}(k)}{2 n}\right. \\
+ & \left.\frac{1}{4 n}\left(\frac{E}{k}-1\right)\left(\frac{2 \rho}{g}\right)^{2}-\frac{1}{120 n^{3}}+\frac{1}{504 n^{5}}\right]
\end{aligned}
$$

Here the Snow formula for the difference between the inductance of a helix of round wire and cylindrical current sheet is expressed in terms of the simple Rosa correction constants $A$ and $B$ (curvature terms omitted). Thus the error of the Rosa correction may be calculated for any desired case.

For the example solved by Snow on page 476, $n=400, g=0.1$, $a=15, \alpha=0.05$. The value of $A$ (see principal term of formula (7)) is -0.13629 , and from Table 3 , for $n=400, B=0.33455$. Thus the simple Rosa correction is $4 \pi n a[0.13629-0.33455]=-14949 \mathrm{~m} \mu \mathrm{h}$ (millimicrohenrys), whereas Snow's equation (114) and formula (34) both give - 14883. Since the value of $L$ is $26,568,401$, this difference of $66 \mathrm{~m} \mu \mathrm{h}$ amounts to about 2.5 parts in $1,000,000$ of the whole

\footnotetext{
The first term of the second line of this equation should read $-\frac{1}{3} \log _{e} \frac{a}{p}$, instead of $-\frac{1}{3} \log _{e} \frac{\alpha}{p}$. The correct reading is employed in the example of p. 476 .

" The difference of 4 parts in $1,000,000$ found by Snow in the solution of the same problem is explained by the use of the value 0.3351 for B, as given in Table 8, B. S. Sci. Paper No. 169. This table has been found to be in error at this and other points as explained in Section V.
} 
inductance, the Rosa method giving too small a value. ${ }^{4}$ This difference is mainly due to the neglect of the axial component of the current. The formula for a continuous helical current sheet is given in Snow's formula.(129). In the nomenclature of the present paper it reads

$$
L_{s}=L_{o}+4 \pi n a\left[\frac{A_{2}(k)}{2 n}-\frac{0.03775}{n}+\frac{1}{6 n}\left[2\left(\frac{K-E}{k}\right)-k K\right]\right]
$$

$K$ and $E$ being the complete elliptical integrals of first and second kind to the modulus, $k$, defined for the preceding equation. The quantity in the brackets of the last term of equation (35) is tabulated in Table I, Bureau of Standards Scientific Paper No. 169, or may also be obtained as $\frac{1000 f}{4 \pi}$, using the values of $f$ given in Table 1, Scientific Paper No. 498.

Subtracting equation (35) from equation (34) there results

$$
\begin{gathered}
L-L_{s}=4 \pi n a\left[-(A+B)-\frac{1}{6 n} \log _{e} \frac{n g}{a}+\frac{0.01322}{n}\right. \\
\left.+\frac{1}{4 n}\left(\frac{E}{k}-1\right)\left(\frac{2 \rho}{g}\right)^{2}-\frac{1}{6 n}\left\{2\left(\frac{K-E}{k}\right)-k K\right\}-\frac{1}{120 n^{3}}+\frac{1}{504 n^{5}}\right]
\end{gathered}
$$

which gives the correction for cross section which has to be applied to the formula for a continuous helical current sheet of tape with negligible insulation to obtain the inductance of a helix of round wire of the same radius, pitch, and number of turns. The error of the simple Rosa correction is given by the terms in equation (36) exclusive of $-4 \pi n a(A+B)$. For the previous example these extra terms are, in order, $4 \pi n a[-0.000409+0.000033+0.000213-0.000024]=$ $-14.1 \mathrm{~m} \mu \mathrm{h}$; that is, the error of the simple Rosa correction when applied to the true helical current sheet (the logical current sheet formula), is only about one-half of 1 part in $1,000,000$ of the total inductance of the coil. If this error be attributed mainly to the neglect of the curvature terms in the Rosa correction (and the algebraic sign is what would be expected), its value shows that the curvature terms are proportionately smaller for the long coil than for the shorter wound on the same form. For example, for a coil of only 30 turns wound with the same pitch on the same form, the correction to the Rosa correction in equation (36) is $-0.55 \mathrm{~m} \mu \mathrm{h}$ in a total of 540,845 ; that is, about 1 part in $1,000,000$. In this case, where the convergence of the formula for the summation of the curvature terms is satisfactory, their calculated value by equations (16) and (18) is $-0.79 \mathrm{~m} \mu \mathrm{h}$.

Before testing the use of the Rosa method for a helix of rectangular wire, it is convenient to generalize the method for a solenoid wound with wire of any cross section. 


\section{GENERALIZATION OF THE ROSA METHOD FOR A SOLENOID WOUND WITH WIRE OF ANY CROSS SECTION}

If we put

$R_{w}=$ the geometric mean distance of the cross section of the wire from itself,

$R_{t}=$ the geometric mean distance of a turn of the current sheet (straight line),

$r_{w p}=$ the geometric mean distance of the cross sections of a pair of wires separated by a distance $p g$,

$r_{t p}=$ the geometric mean distance of the sections of the corresponding turns of the current sheet, $L_{s}=$ inductance of the equivalent current sheet,

then the general expressions for the Rosa corrections are

and

$$
\begin{aligned}
-4 \pi n a A & =-4 \pi n a\left(\log _{e} R_{w}-\log _{e} R_{t}\right) \\
-4 \pi n a B & =4 \pi n a\left[\frac { 2 } { n } \left\{(n-1) \log _{e} \frac{r_{t 1}}{r_{w 1}}+(n-2) \log _{e} \frac{r_{t 2}}{r_{w 2}}+\cdots\right.\right. \\
& \left.\left.+(n-p) \log _{e} \frac{r_{t p}}{r_{w p}}+\cdots+\log _{e} \frac{r_{t(n-1)}}{r_{w(n-1)}}\right\}\right]
\end{aligned}
$$

$$
L=L_{s}-4 \pi n a(A+B)
$$

If the cross section of the wire is not circular, it is convenient to write the second of these equations in the form

$$
-4 \pi n a B=4 \pi n a\left[\frac{2}{n} \sum_{p=1}^{n-1}(n-p) \log _{e} \frac{r_{t p}}{p g}-\frac{2}{n} \sum_{p=1}^{n-1}(n-p) \log _{e} \frac{r_{w p}}{p g}\right]
$$

The first series of terms gives the $B$ correction for circular wire and is tabulated in Table 3. The second series depends upon the differences of the geometric mean distances of the actual cross sections of the wire and those of circular turns wound with the same pitch and number of turns. In general, formulas for the geometric mean distance are not known, except in the important case of a rectangular cross section. The comparison of the Rosa correction with Snow's formula for wire of rectangular cross section is therefore of special interest. The author has in preparation tables for aiding in numerical computations for this case. 
IX. COMPARISON OF THE ROSA METHOD WITH SNOW'S FORMULA FOR THE INDUCTANCE OF A HELIX OF WIRE OF RECTANGULAR CROSS SECTION

Snow's formula (126) for the inductance of a helix of wire of rectangular cross section of axial dimension $\beta$ and radial thickness $a$ is as follows for uniform current density over the cross section:

$$
\begin{aligned}
L & =L_{o}+2 \pi a\left\{A_{2}(k)+\frac{1}{3}\left[1+\frac{\alpha^{2}-\beta^{2}}{g^{2}}\right] \log _{e} \frac{2 \pi a}{g}\right. \\
& -2 n\left[-\log _{e} \frac{g}{p}-4.41212+\frac{1}{6}\left(\tan ^{2} \theta \log _{e} \sin \theta+\cot ^{2} \theta \log _{e} \cos \theta\right)\right. \\
& +\frac{2}{3}\left(\left(\frac{\pi}{2}-\theta\right) \tan \theta+\theta \cot \theta\right) \\
& \frac{f\left(\frac{\alpha}{g}, \frac{\beta}{g}\right)-f\left(\frac{\alpha}{g}, o\right)-f\left(0, \frac{\beta}{g}\right)}{6\left(\frac{\alpha}{g}\right)^{2}\left(\frac{\beta}{g}\right)^{2}} \\
& \left.-\frac{8}{\sin ^{2} \theta \cos ^{2} \theta} \sum_{m=1}^{\infty} \frac{\left(\frac{\rho}{g}\right)^{2 m}\left(\cos ^{2 m+4} \theta+(-1)^{m} \sin ^{2 m+4} \theta\right.}{2 m(2 m+1)(2 m+2)(2 m+3)(2 m+4)}\left(S_{2 m}-1\right)\right] \\
& \frac{2}{3}\left(\frac{E}{k}-1\right)\left(\frac{\alpha}{g}\right)^{2}+\frac{1}{3}\left[1.18123+2\left(\frac{K-E}{k}\right)-k K\right] \frac{\beta^{2}-\alpha^{2}}{g^{2}} \\
& -\frac{25}{3}-\frac{f\left(\frac{\alpha}{g}, \frac{\beta}{g}\right)^{2}-f\left(\frac{\alpha}{g}, o\right)-f\left(0, \frac{\beta}{g}\right)}{3\left(\frac{\alpha}{g}\right)^{2}\left(\frac{\beta}{g}\right)^{2}} \\
& \left.-\frac{16}{\sin ^{2} \theta \cos ^{2} \theta} \sum_{m=2}^{\infty} \frac{\left(\frac{\rho}{g}\right)^{2 m}\left(\cos ^{2 m+4} \theta+(-1)^{m} \sin ^{2 m+4} \theta\right.}{2 m(2 m+1)(2 m+2)(2 m+3)(2 m+4)}\left(S_{2 m-1}-1\right)\right\}
\end{aligned}
$$

In this equation $\theta=\tan ^{-1} \frac{\alpha}{\beta}, S_{m}=\sum_{k=1}^{\infty} \frac{1}{k^{m}}$ and the $f^{\prime}$ 's are functions of the ratios $\frac{\alpha}{g}$ and $\frac{\beta}{g}$, defined in Snow's formulas (123), (124), and (125). For the rest the equation has been written in terms of quantities already defined.

Making use of the definition of the geometric mean distance of a rectangle in terms of $\theta$, Snow's equation (118), and the known expression for the geometric mean distance of a straight line $R_{t}=\log g-\frac{3}{2}$, and the defining equation for $A$ in equation (37), the second and third lines of equation (39) may be written

$$
4 \pi n a\left[-A+\left(\frac{3}{2}-\log _{e} 2 \pi\right)+\frac{25}{6}\right]
$$


Comparing the defining equations for the $f$ functions with the formula for the geometric mean distance of two equal parallel rectangles (12) it is seen that the fourth line of equation (39) is

$$
4 \pi n a\left[-2 n \log _{e} \frac{r_{w 1}}{g}-\frac{25}{6} n\right]
$$

while the next to last line- of equation (39) is

$$
4 \pi a\left[2 \log _{e} \frac{r_{w 1}}{g}\right]
$$

so that together they give the first term of the last series of terms in equation (38).

The geometric mean distance of two equal parallel rectangles may be expanded in a series which holds for separations, $p g$, which are greater than the dimensions of the rectangle. The series is

$$
\begin{aligned}
\log _{e} \frac{r_{w p}}{p g} & =-\frac{1}{12}\left(\frac{\beta}{p g}\right)^{2}-\frac{1}{60}\left(\frac{\beta}{p g}\right)^{4}-\frac{1}{168}\left(\frac{\beta}{p g}\right)^{6}-\frac{1}{360}\left(\frac{\beta}{p g}\right)^{8}-\cdots \\
& +\frac{1}{12}\left(\frac{\alpha}{p g}\right)^{2}\left[1+\frac{1}{2}\left(\frac{\beta}{p g}\right)^{2}+\frac{1}{3}\left(\frac{\beta}{p g}\right)^{4}+\frac{1}{4}\left(\frac{\beta}{p g}\right)^{6}+\cdots\right] \\
& -\frac{1}{60}\left(\frac{\alpha}{p g}\right)^{4}\left[1+\frac{5}{3}\left(\frac{\beta}{p g}\right)^{2}+\frac{7}{3}\left(\frac{\beta}{p g}\right)^{4}+\cdots\right]+\frac{1}{168}\left(\frac{\alpha}{p g}\right)^{6}[1 \\
& \left.+\frac{7}{2}\left(\frac{\beta}{p g}\right)^{2}+\cdots\right]-\frac{1}{360}\left(\frac{\alpha}{p g}\right)^{8}+\cdots
\end{aligned}
$$

Expanding the summation in the fifth line of Snow's equation and comparing the result with equation (43), it is seen that the Snow series is identical with the terms $\sum_{2}^{n-1} n \log _{e} \frac{r_{w p}}{p g}$ in equation (38), except that the series $S_{2 m}$ are taken to infinity, whereas in the summation of the geometric mean distances it ends with $(n-1)$. The difference, that is, the sum between $(n-1)$ and infinity may, however, readily be expressed in a series involving powers of $\frac{1}{n}$. The series in the last line of equation (39) is identical with the terms $\sum_{2}^{n-1} p \log _{e} \frac{r_{w p}}{p g}$, except that the second degree terms in $\alpha$ and $\beta$ are lacking and that here again the summations $S_{2 m-1}$ are carried to infinity instead of $(n-1)$, and it will be necessary to expand the difference in a series in powers of $\frac{1}{n}$. 
The remaining terms in $\sum_{2}^{n-1} p \log _{e} \frac{r_{w p}}{p g}$ in the $B$ (rectangle) correction of equation (38) may be identified with the last term in the first line of equation (39) (that in $\left(\alpha^{2}-\beta^{2}\right)$ ), by writing it to involve $\log n g$ and substituting for $\log n$ its value in terms of the series $\sum_{2}^{n-1} \frac{1}{k}$ which arises when the series of equation (43) is used for $\log \frac{r_{w p}}{p g}$.

The remaining term in $\log \frac{2 \pi a}{g}$ in the first line of equation (39) may be expressed in terms of the Rosa $B$ correction for circles, as was done in Section VII.

The results of collecting all the transformed terms is that Snow's equation (39) may be written in the form

$$
\begin{aligned}
L & =L_{0}+4 \pi n a\left[-(A+B)-\frac{1}{6 n} \log _{e} \frac{n g}{a}-\frac{0.02453}{n}-\frac{1}{120 n^{3}}+\frac{1}{504 n^{5}}\right. \\
& +\frac{A_{2}}{2 n}+\frac{1}{3 n}\left(\frac{E}{k}-1\right)\left(1+\frac{2 a u_{x}}{u}\right)\left(\frac{\alpha}{g}\right)^{2} \\
& +\frac{1}{6 n} \frac{\left(\beta^{2}-\alpha^{2}\right)}{g^{2}}\left\{\log _{e} \frac{n g}{a}-0.07943+2 \frac{(K-E)}{k}-k K+\frac{1}{12 n^{2}}-\frac{1}{40 n^{4}}\right\} \\
& -\frac{1}{3 n^{3}}\left(1-\frac{1}{2 n^{2}}\right)\left(\frac{1}{60} \frac{\beta^{4}}{g^{4}}-\frac{1}{24} \frac{\beta^{2} \alpha^{2}}{g^{4}}+\frac{1}{60} \frac{\alpha^{4}}{g^{4}}\right) \\
& \left.-\frac{1}{10 n^{5}}\left(\frac{1}{168} \frac{\beta^{6}}{g^{6}}-\frac{1}{36} \frac{\beta^{4} \alpha^{2}}{g^{6}}+\frac{1}{36} \frac{\beta^{2} \alpha^{4}}{g^{6}}-\frac{1}{168} \frac{\alpha^{6}}{g^{6}}\right)+\cdots\right]
\end{aligned}
$$

where $A$ and $B$ are defined in equations (37) and (38).

For square wire, the second and sixth degree terms in $\frac{\alpha}{g}$ and $\frac{\beta}{g}$ drop out, and the fourth degree terms become $\frac{1}{360 n^{3}}\left(1-\frac{1}{2 n^{2}}\right) \frac{\beta^{4}}{g^{4}}$. For the special case $\alpha=0$ and $\beta=g$, equation (44) goes over into Snow's formula for a continuous helical current sheet, as it should. It is of interest to note that in that case the terms in powers of $\frac{1}{n}$ all cancel, thus giving a check on the coefficients in the series expansions, and especially furnishing evidence that the term in $\frac{1}{n^{5}}$ in formula (31) is correct.

Making use of the formula (44) the difference may be evaluated between the accurate formula of Snow for square wire and the solution by Rosa's method in the case of the example treated by Snow 
on page 477 of his article. In this the coil is supposed to be wound with square wire, instead of round, the side of the section being taken as $\alpha=\beta=0.05 \mathrm{~cm}$. The radius, number of turns, and pitch of the winding are taken the same as in the, preceding problem for round wire. The distribution of the current over the cross section being assumed to be uniform, the value of $u_{x}$ in equation (44) is zero.

For the calculation of the $A$ correction, we have $\log R_{t}=\log g-\frac{3}{2}$, and $R_{2}=0.44705 \alpha$. Thus from equation (37), $-A=\log \frac{R_{t}}{R_{w}}=-0.001773$. The $B$ correction for the circle is 0.33455 as before. For the value of $\log \frac{r_{w 1}}{g}$ the exact formula gives 0.0005109 , which multiplied by $\frac{2}{n}(n-1)$ amounts to 0.001019 . For the more distant wires, the formula (43) may be used, and only a few terms have to be included. These bring up the total of the value of $B$ for the rectangles to 0.001104 . Thus the Rosa terms in equation (44) amount to

$$
-0.001773-0.334554-0.001104=-0.337431
$$

which, multiplied by $4 \pi n a$, gives for the Rosa correction $-25442 \mathrm{~m} \mu \mathrm{h}$. The correction terms in equation (44) are found to be

$$
\begin{aligned}
\frac{A_{2}}{2 n} & =0.001125 \\
-\frac{1}{6 n} \log _{e} \frac{n g}{a} & =-0.000409 \\
-\frac{0.02453}{n} & =-0.000061 \\
\frac{1}{3 n}\left(\frac{E}{k}-1\right)\left(\frac{\alpha}{g}\right)^{2} & =\frac{0.000284}{0.000939}
\end{aligned}
$$

which, multiplied by $4 \pi n a$, show that the error of the Rosa correction is $70.8 \mathrm{~m} \mu \mathrm{h}$. This amounts to about 2.7 parts in $1,000,000$ of the whole inductance. Thus, the true correction for cross section to be applied to the inductance of the cylindrical current sheet, in order to find the inductance of the helical coil of square wire, is $-25442+71$ $=-25371 \mathrm{~m} \mu \mathrm{h}$. This value, found by equation (44), should agree with the value found directly from Snow's equation (126). The value calculated by Snow is -25345 . The source of the discrepancy lies in an error in the value of the term multiplied by $\frac{8}{3}$ in the last equation of the calculation on page 477 . The true value is 1.56288 instead 
of 1.56268 , as given by Snow. Making this change, Snow's equation gives -25372 .

The error of the Rosa method quoted in Snow's paper for this problem is 6 parts in $1,000,000$. This is affected by both the error in the value of $B$ used for the circle (already discussed in Section V) and by the numerical error in Snow's calculation just noted.

If the Rosa corrections were applied to the formula of Snow for a continuous helical current sheet we use the result from equation (35), that the inductance $L_{s}$ of the helical current sheet is related to $L_{o}$, that of the cylindrical current sheet, by the relation $L_{s}=L_{0}+79.5$ $\mathrm{m} \mu \mathrm{h}$, and the calculated inductance of the coil is

$$
L=L_{o}+79.5-25442=L_{o}-25362 \mathrm{~m} \mu \mathrm{h} .
$$

which is in error by only 0.4 of 1 part in $1,000,000$. This is in line with the calculation on page 181, which showed that the error of the Rosa correction as ordinarily applied to the cylindrical sheet formula is principally due to neglect of the helicity. The residual error of the Rosa method is practically the same for both round and square cross section, as would be expected.

\section{CONCLUSION}

In the preceding sections it has been shown that the simple Rosa method of correction for the effect of cross section gives results for circular solenoids of round or rectangular wire which are amply accurate for all practical cases. Indeed, for the examples given, the Rosa method gives results of high precision, when applied to the logical current sheet formula-that in which the axial component of magnetic field is taken into account. The numerical proof of this degree of accuracy in the Rosa method is made possible by the existence of the accurate formulas of Snow.

Unfortunately, formulas corresponding to those of Snow for other cases, circular flat spirals, polygonal solenoids, and polygonal spirals, are not available, and it would seem to be a work of great difficulty to derive them. Lacking them, the Rosa method applied to the available current sheet formulas offers the simplest and most accurate general method of calculation. It has been shown that for short windings the summation method and the Rosa method are in agreement, but the Rosa method applies to any case where the current sheet formula is available. It remains to note the use of the method in the cases already cited.

It is easy to show that for a circular flat spiral the curvature terms in the Rosa correction are of second degree in $\frac{g^{2}}{a^{2}}$ and may, therefore, be neglected as of less importance than the effect of the radial component of the current. The current sheet formula for a disk-shaped or annular current sheet of negligible axial thickness is known, as 
well as series expansions holding for the case where the axial width of the current sheet is small compared with the mean radius (18). In all of these the radial component of the current is neglected. Thus the inductance of a flat circular spiral wound with wire of round or rectangular cross section may best be found by applying the simple Rosa correction, calculated by the methods and formulas already fully treated, to the inductance of a current sheet having the same mean radius as the spiral coil and a radial width equal to the product of the pitch by the number of turns.

The case of a short polygonal solenoid has already been fully treated and the inductance formula given as equation (25). In this it is to be noted that the principal terms in the correction equations here are the Rosa constants; that is, for round wire the simple Rosa constants are the same for polygonal solenoids as for circular. In both cases they are multiplied by twice the length of wire in the winding. No formula is available for long polygonal solenoids, except in the case of the square solenoids (14). It would be possible, though a great deal of work, to derive the formulas for the other cases, but it is sufficient in practical cases to make use of the formula for the equivalent circular cylindrical current sheet as was described (13) in Bureau of Standards Scientific Paper No. 468, and to apply to this the simple Rosa correction.

The calculation of the inductance of a polygonal spiral is covered by formulas (26) and (27). These apply most accurately to spirals where the radial width is small compared with the side of the polygon, but this will cover many practical cases. No general formula is known for a disk of polygonal shape. A good approximation to it can be obtained by calculating the inductance of a circular disk current sheet whose mean element incloses the same area as the mean element of the polygonal disk. It is to be noticed that in equation (27) the principal Rosa constants are the same as those which hold for circular and polygonal solenoids of round wire.

\section{BIBLIOGRAPHY}

1. Chester Snow, Formula for the Inductance of a Helix Made with Wire of Any Section, B. S. Sci. Paper No. 537. In this, formula (114) gives the inductance of a helix of round wire, formula (126) that of a helix of wire of rectangular cross section, and formula (129) the inductance of a continuous helical current sheet.

2. F. W. Grover, Methods for the Derivation and Expansion of Formulas for the Mutual Inductance of Coaxial Circles and for the Inductance of Single-Layer Solenoids, B. S. Jour. Research, 1 (RP16), p. 487; October, 1928.

3. Strasser, A Formula for the Inductance of a Single-Layer Coil of Round Wire by the Summation Method, Wied. Ann., 1\%, p. 763; 1905. A somewhat more accurate form of Strasser's formula is given in B. S. Sci. Paper No. 169 , p. 124 ; 1912; formula (82), and a table of Strasser's constants in Table 5, p. 195 . Note that in the present paper $A_{1}$ and $B_{1}$ correspond to $A$ and $B$ in Strasser's notation. 
4. A. Esau, A Summation Formula for the Inductance of a Flat Circular Spiral, Jahrb. der drahtl. Tel., 5, p. 212; 1911. This is modeled on Strasser's formula for the solenoid. Ueber die Berechnung des Selbstinduktionskoefficienten von Spulen mit rechteckigem bzw. quadratischem Querschnitt. I. Nebeneinander liegende Windungen, Jahrb. der drahtl, Tel., 14, p. 271; 1919. II. Spulen mit uebereinander liegenden Windungen (Flachspulen), Jahrb. der drahtl. Tel., 14, p. 386; 1919.

5. I. Koga, Inductance of Polygonal Coils, J. Inst. of E. E. of Japan; October, 1924. Formulas for triangular, hexagonal, and octagonal solenoids and spirals. Gives an asymptotic formula for $A_{1}$ on page 7 .

6. E. B. Rosa, Calculation of the Self-Inductance of Single-Layer Coils, B. S. Bulletin, 2, p. 161; 1906. Rosa's formula is also given in B. S. Sci. Paper No. 169 , p. 122 , as formula (80) and Tables 7 and 8, pp. 197-199, give his tables of constants $A$ and $B$.

7. Yasujiro Niwa, A study of Coils Wound on Rectangular Frames with Special Reference to the Calculation of the Inductance, Researches of the Electrotechnical Laboratory, Tokyo, No. 141, April, 1924. A very full collection of formulas for the self and mutual inductance of various rectangular current sheets, together with tables of constants and curves. On page 56 is given the Rosa correction for a square solenoid of round wire.

8. A. Esau, Criticism of Niwa's Formula for the Inductance of Square Solenoids, Jahrb. der drahtl. Tel., 18, p. 453; 1921. This is answered by Niwa, pp. 51-55 of the reference in No. 7.

9. M. Wien, The Inductance of a Circular Ring of Wire of Circular Cross Section, Wied. Ann., 53, p. 935; 1894. Given as formula (61) of B. S. Sci. Paper No. 169, p. 110.

10. Maxwell, Series Formula for the Mutual Inductance of Equal Coaxial Circular Filaments, Elect. and Mag., II, sec. 705. Also appears as formula (12), p. 13 of B. S. Sci. Paper No. 169.

11. Rayleigh and Niven, Inductance of a Short Cylindrical Current Sheet, Proc. Roy. Soc., 32, pp. 104-141; 1881. Rayleigh's Collected Papers, 2, p. 15. This also appears as formula (69) of B. S. Sci. Paper No. 169.

12. Formulas for the calculation of the geometric mean distance of certain figures were given by Maxwell, Elect. and Mag., II, secs. 691 and 692; Gray, Absolute Measurements, II, pt. 1, pp. 288-306; Rosa, On the Geometric Mean Distances of Rectangular Areas and the Calculation of Self-Inductance, B. S. Bulletin, 3, p. 1; 1907. The more important of these are collected in B. S. Sci. Paper No. 169, pp. 167-170, formulas (123) to (131), inclusive.

13. F. W. Grover, Formulas and Tables for the Calculation of the Inductance of Coils of Polygonal Form, B. S. Sci. Paper No. 468; $1923 . \quad$ Triangular square, hexagonal, and octagonal solenoids. Exact formula for square solenoid on p. 742. Radius of equivalent circular solenoid, p. 753 and Table 1, p. 761 .

14. Y. Niwa, On the Solenoid with Rectangular Cross Section, Researches of the Electrotechnical Laboratory, Tokyo, No. 73; November, 1918; and J. Elect. Soc. Japan, pp. 535-564; 1919.

15. Formulas for Arithmetic Mean Distances and for Arithmetic Mean Square Distances were Given by Rosa, B. S. Bulletin, 4, p. 326; 1907 . These are collected pp. 171-172, B. S. Sci. Paper No. 169. Of these formulas (137) and (138) refer to straight lines.

16. Bruno Rolf, Zur Frage der Berechnung des Selbstinduktionskoefficienten von Spulen mit quadratischem Windungsquerschnitt, Jahrb. der drahtl. Tel., 19, p. 127, 1922. He gives an asymptotic formula for $A_{1}$ and simplifies Esau's formula for the inductance of a square solenoid so as to admit of 
tabulation for any number of turns. This does not take into account that Esau's formula, like other summation formulas, converges only for short coils.

17. Stirling's formula, Smithsonian Math. Tables, p. 28. Euler summation formula, Smithsonian Math. Tables, p. 25.

18. Spielrein, Arch. für Elektrotech., 3, p. 187, 1915; gives a formula for the inductance of an annular disk current sheet. See also B. S. Sci. Paper No. 320 , p. $555 ; 1918$; formula $(24 \mathrm{~A})$. A series formula for the same case is given by T. Lyle, Phil. Trans., 213A, pp. 421-435; 1914; and B. S. Sci. Paper No. 320, p. 557; 1918; and Tables C and D, pp. 569-570. In a paper On the Inductance of Single-Layer Flat Coils, S. Butterworth, Proc. Lond. Phys. Soc., 32, pt. 1, p. 31; December, 1919; are derived formulas which check those of Lyle and Spielrein.

19. Revision of the Formulae of Weinstein and Stefan for the Mutual Inductance of Coaxial Coils, B. S. Bulletin, 2, p. 331; 1906. The revised Stefan formula appears as equation (48) and the revised Weinstein formula as equation (50) in B. S. Sci. Paper No. 169.

\section{APPENDIX}

TABLE 3.-Values of the Rosa constant $B$, calculated by formula (31)

\begin{tabular}{|c|c|c|c|c|c|}
\hline$n$ & $B$ & $n$ & $B$ & $n$ & $B$ \\
\hline $\begin{array}{l}1 \\
2 \\
3 \\
4 \\
5\end{array}$ & $\begin{array}{l}0 \\
.11371 \\
.16626 \\
.19728 \\
.21800\end{array}$ & $\begin{array}{l}31 \\
32 \\
33 \\
34 \\
35\end{array}$ & $\begin{array}{r}\text { 0. } 30874 \\
.30949 \\
.31019 \\
.31086 \\
.31149\end{array}$ & $\begin{array}{l}100 \\
110 \\
120 \\
130 \\
140\end{array}$ & $\begin{array}{r}0.32689 \\
.32775 \\
.32847 \\
.32909 \\
.32963\end{array}$ \\
\hline $\begin{array}{r}6 \\
7 \\
8 \\
9 \\
10\end{array}$ & $\begin{array}{l}.23293 \\
.24426 \\
.25318 \\
.26042 \\
.26641\end{array}$ & $\begin{array}{l}36 \\
37 \\
38 \\
39 \\
40\end{array}$ & $\begin{array}{r}.31210 \\
.31267 \\
.31322 \\
.31374 \\
.31424\end{array}$ & $\begin{array}{l}150 \\
160 \\
170 \\
180 \\
190\end{array}$ & $\begin{array}{r}33010 \\
.33052 \\
.33090 \\
.33123 \\
.33153\end{array}$ \\
\hline $\begin{array}{l}11 \\
12 \\
13 \\
14 \\
15\end{array}$ & $\begin{array}{r}27146 \\
.27579 \\
.27954 \\
.28282 \\
.28573\end{array}$ & $\begin{array}{l}41 \\
42 \\
43 \\
44 \\
45\end{array}$ & $\begin{array}{l}.31471 \\
.31517 \\
.31561 \\
.31602 \\
.31643\end{array}$ & $\begin{array}{l}200 \\
220 \\
240 \\
260 \\
280\end{array}$ & $\begin{array}{r}33181 \\
.33229 \\
.33269 \\
.33304 \\
.33334\end{array}$ \\
\hline $\begin{array}{l}16 \\
17 \\
18 \\
19 \\
20\end{array}$ & $\begin{array}{l}.28832 \\
.29064 \\
.29273 \\
.29464 \\
.29637\end{array}$ & $\begin{array}{l}46 \\
47 \\
48 \\
49 \\
50\end{array}$ & $\begin{array}{r}31681 \\
.31718 \\
.31754 \\
.31789 \\
.31822\end{array}$ & $\begin{array}{l}300 \\
350 \\
400 \\
450 \\
500\end{array}$ & $\begin{array}{r}.33360 \\
.33414 \\
.33455 \\
.33488 \\
.33514\end{array}$ \\
\hline $\begin{array}{l}21 \\
22 \\
23 \\
24 \\
25\end{array}$ & $\begin{array}{r}.29796 \\
.29942 \\
.30077 \\
.30202 \\
.30318\end{array}$ & $\begin{array}{l}55 \\
60 \\
65 \\
70 \\
75\end{array}$ & $\begin{array}{l}.31972 \\
.32099 \\
.32208 \\
.32304 \\
.32387\end{array}$ & $\begin{array}{l}550 \\
600 \\
650 \\
700 \\
750\end{array}$ & $\begin{array}{r}.33536 \\
.33555 \\
.33571 \\
.33584 \\
.33596\end{array}$ \\
\hline $\begin{array}{l}26 \\
27 \\
28 \\
29 \\
30\end{array}$ & $\begin{array}{l}.30427 \\
.30528 \\
.30623 \\
.30712 \\
.30795\end{array}$ & $\begin{array}{r}80 \\
85 \\
90 \\
95 \\
100\end{array}$ & $\begin{array}{l}.32461 \\
.32527 \\
.32587 \\
.32640 \\
.32689\end{array}$ & $\begin{array}{r}800 \\
850 \\
900 \\
950 \\
1000 \\
\infty\end{array}$ & $\begin{array}{r}.33607 \\
.33616 \\
.33625 \\
.33633 \\
.33640 \\
.33788\end{array}$ \\
\hline
\end{tabular}

Values not found in the table may be calculated by the formula

$$
B=0.337877-\frac{1}{6 n} \log _{e} n-\frac{0.330842}{n}-\frac{1}{120 n^{3}}+\frac{1}{504 n^{5}}
$$

WASHINGTON, January 25, 1929. 